JOURNAL OF THE AMERICAN MATHEMATICAL SOCIETY

Volume 18, Number 4, Pages 763-778

S 0894-0347(05)00492-3

Article electronically published on June 10, 2005

\title{
THE LOEWNER DIFFERENTIAL EQUATION AND SLIT MAPPINGS
}

\author{
DONALD E. MARSHALL AND STEFFEN ROHDE
}

\section{INTRODUCTION AND RESULTS}

In his study of extremal problems for univalent functions, K. Löwner 11] (who later changed his name into C. Loewner) introduced the differential equation named after him. It was a key ingredient in the proof of the Bieberbach conjecture by de Branges [2]. It was used by L. Carleson and N. Makarov in their investigation of a process similar to DLA 3. Recently, O. Schramm 20] found a description of the scaling limits of some stochastic processes in terms of the Loewner equation (assuming the validity of some conjectures such as existence of the limits). This led him to the definition of a new stochastic process, the "Stochastic Loewner Evolution" (SLE). The SLE has been further explored in the work of Lawler, Schramm and Werner (see [14] and the references therein) and led to a proof of Mandelbrot's conjecture about the Hausdorff dimension of the Brownian frontier. The SLE has also played a crucial role in S. Smirnov's 21] recent work on percolation.

Somewhat surprisingly, the geometry of the solutions to the Loewner equation is not very well understood. This paper addresses the regularity of solutions in the deterministic setting.

Let $\Omega_{t}, t_{0} \leq t \leq t_{1}$, be a continuously increasing sequence of simply connected planar domains, and let $z_{0} \in \bigcap_{t} \Omega_{t}$. Then there are conformal maps $f_{t}: \mathbb{D} \rightarrow \Omega_{t}$ of the unit disc with $f_{t}(0)=z_{0}$ and $f_{t}^{\prime}(0)>0$. The continuity of the domain sequence can be expressed by saying that the map $t \mapsto f_{t}$ is continuous in the topology of locally uniform convergence. Since $f_{t}^{\prime}(0)$ is increasing in $t$, we may assume (by reparametrizing $\Omega_{t}$ if necessary) that $f_{t}^{\prime}(0)=e^{t}$ for $t_{0} \leq t \leq t_{1}$. The family $f_{t}, t_{0} \leq t \leq t_{1}$, is called a (normalized) Loewner chain. The Loewner differential equation

$$
\partial_{t} f(z, t)=z p(z, t) \partial_{z} f(z, t)
$$

describes the evolution of a (normalized) Loewner chain $f_{t}(z)$, where $p=p(z, t)$ is analytic in $z$ and has positive real part (see [17]). A particularly important case is the case of slit domains

$$
\Omega_{t}=\Omega \backslash \gamma\left[t, t_{1}\right]
$$

Received by the editors July 1, 2003.

2000 Mathematics Subject Classification. Primary 30C45, 30C20; Secondary 30C62, 30C30.

Key words and phrases. Conformal maps, harmonic measure, quasiconformal maps, quasiarc, conformal welding, Loewner's differential equation, Lipschitz continuous, Hölder continuous.

The authors were partially supported by NSF grants DMS-9800464, DMS-9970398, and DMS0201435.

(C)2005 American Mathematical Society Reverts to public domain 28 years from publication 
where $\Omega \subseteq \mathbb{C}$ is simply connected and $\gamma$ parametrizes a simple arc that is contained in $\Omega$ except for one endpoint $\gamma\left(t_{1}\right) \in \partial \Omega$. In this case, $p$ has a particularly simple form and (1.1) becomes

$$
\partial_{t} f=z \frac{\lambda(t)+z}{\lambda(t)-z} \partial_{z} f
$$

where $\lambda(t)=f_{t}^{-1}(\gamma(t)) \in \mathbb{T}=\partial \mathbb{D}$ (see [5], Chapter 3.3). It is known that $\lambda(t)$ is continuous, and conversely that (1.3) generates a sequence of increasing domains whenever $\lambda$ is continuous. In fact, even (1.1) generates conformal maps onto increasing domains assuming only measurability of $p$ in $t$; see [17]. The paper [13] contains a topological characterization of increasing domain families $\Omega_{t}$ for which (1.1) simplifies to (1.3) with continuous $\lambda$.

It is known [9] that the domains $\Omega_{t}$ obtained from (1.3) are not necessarily slit domains if $\lambda$ is only assumed to be continuous. The main result of this paper is a rather sharp condition on $\lambda$ that guarantees slit domains.

We will first consider (1.2) with $\Omega=\mathbb{C}$, so that $t_{1}=\infty$. A quasiarc is the image of $[0, \infty)$ under a quasiconformal homeomorphism of $\mathbb{C}$. Piecewise smooth arcs without zero angle cusps are quasiarcs, as well as for instance the van Koch snowflake. A simple geometric characterization of quasiarcs is the Ahlfors three-point condition (the diameter of any subarc is bounded above by a bounded multiple of the distance between the endpoints). Denote by $\operatorname{Lip}\left(\frac{1}{2}\right)$ the space of Hölder continuous functions $\lambda$ with exponent $1 / 2,|\lambda(s)-\lambda(t)| \leq c|s-t|^{1 / 2}$, and let $\|\lambda\|_{\frac{1}{2}}$ denote the smallest such $c$.

Theorem 1.1. If $\gamma$ is a quasiarc, then $\lambda \in \operatorname{Lip}\left(\frac{1}{2}\right)$. Conversely, there is a constant $C>0$ such that if $\lambda \in \operatorname{Lip}\left(\frac{1}{2}\right)$ with $\|\lambda\|_{\frac{1}{2}}<C$, then $\mathbb{C} \backslash f_{t}(\mathbb{D})$ is a quasiarc for all $t$.

If the assumption $\|\lambda\|_{\frac{1}{2}}<C$ is replaced by the slightly weaker local condition

$$
\inf _{\epsilon>0} \sup _{|t-s|<\epsilon} \frac{|\lambda(t)-\lambda(s)|}{\sqrt{|t-s|}}<C,
$$

then $\mathbb{C} \backslash f_{t}(\mathbb{D})$ is still a quasiarc. This follows from Lemma 2.7] The assumption $\|\lambda\|_{\frac{1}{2}}<C$ cannot be omitted: we show that there is a function $\lambda \in \operatorname{Lip}\left(\frac{1}{2}\right) \operatorname{such}$ that $\partial \Omega_{t}$ is not even locally connected (and $\Omega_{t} \nearrow \Omega=\mathbb{C}$ ).

By means of a simple transformation, the Loewner equation becomes an ODE: for $t \leq s$, set

$$
\phi_{s, t}=f_{s}^{-1} \circ f_{t}
$$

Then $\phi_{s, t}$ maps $\mathbb{D}$ into $\mathbb{D}$, satisfies $\phi_{s, t}(0)=0$ and $\phi_{s, t}^{\prime}(0)=e^{t-s}$. By differentiating the identity $f_{s} \circ \phi_{s, t}=f_{t}$ with respect to $s$, (1.3) becomes

$$
\partial_{s} \phi_{s, t}(z)=-\phi_{s, t}(z) \frac{\lambda(s)+\phi_{s, t}(z)}{\lambda(s)-\phi_{s, t}(z)} \text {. }
$$

Notice that $\phi_{t, t}=\mathrm{id}$. Fix $t$ and set $g_{s}=\phi_{t+s, t}$ for $0 \leq s \leq s_{0}:=t_{1}-t$, and let $\xi(s)=\lambda(t+s)$. Then

$$
\partial_{s} g_{s}=-g_{s} \frac{\xi(s)+g_{s}}{\xi(s)-g_{s}} \quad \text { for } \quad s \in\left[0, s_{0}\right), \quad g_{0}=\mathrm{id} .
$$

Notice that the solutions to the initial value problem (1.5) are unique, whereas the solutions to (1.1) and (1.3) are unique only up to post-composition with a conformal map of $\bigcup_{t} f_{t}(\mathbb{D})$, simply because no initial conditions are imposed (if $t_{1}=\infty$, a 
certain terminal condition is automatically satisfied and hence solutions are unique in this case). Therefore it is more natural to work with (1.5). Theorem 1.1 is a simple consequence of

Theorem 1.2. Let $\xi:\left[0, s_{0}\right] \rightarrow \partial \mathbb{D}$ be continuous and $g_{s}$ the solution to (1.5). If $g_{s_{0}}(\mathbb{D})$ is a quasislit-disc, then $\xi \in \operatorname{Lip}\left(\frac{1}{2}\right)$. Conversely, if $\|\xi\|_{\frac{1}{2}}<C$, then $g_{s}(\mathbb{D})$ is a quasislit-disc for all $s$.

A quasislit-disc is, by definition, the image of a radially slit disc under a quasiconformal map fixing $\mathbb{D}$ and 0. See Section 2 for basic properties. Theorems 1.1 and 1.2 are quantitative in the sense that the quasiconformal constant $K$ and the $\operatorname{Lip}\left(\frac{1}{2}\right)$-norm depend on each other, and $K$ is close to 1 if and only if $\|\xi\|_{\frac{1}{2}}$ is close to 0 .

The idea of the proof of Theorem 1.2 is as follows: The Loewner equation can be viewed as a decomposition of a conformal map $f$ into infinitesimal conformal factors. This suggests approximating $f$ by a composition of (finitely many) conformal maps. Given $\xi \in \operatorname{Lip}\left(\frac{1}{2}\right)$, it is not hard to approximate $\xi$ by functions $\xi^{(\varepsilon)} \in \operatorname{Lip}\left(\frac{1}{2}\right)$ for which it is easy to see that the solutions $g_{s}^{(\varepsilon)}$ generate slits. If $\left\|\xi^{(\varepsilon)}\right\|_{\frac{1}{2}}$ is small, then the conformal welding in these slits can be shown to be quasisymmetric, by analyzing (1.5) on $\mathbb{T}$. This easily implies that the slits are quasiconformal slits. By compactness of the space of quasislit-domains, one obtains quasislits in the limit $\varepsilon \rightarrow 0$.

In (1.1) - (1.5), $f_{t}$ and $g_{s}$ are normalized in an interior point. A different important normalization requires $f$ or $g$ to fix a boundary point. Then it is more convenient to consider the upper half-plane $\mathbb{H}$ instead of $\mathbb{D}$, and to choose $\infty$ as this boundary point. The normalization $f^{\prime}(t)=e^{t}$ is then to be replaced by the hydrodynamic normalization $f_{t}(z)=z-2 t / z+O\left(1 / z^{2}\right)$ as $z \rightarrow \infty(t \geq 0)$, and the equation corresponding to (1.5) is

$$
\partial_{s} g_{s}=\frac{2}{\xi(s)-g_{s}} \quad \text { for } \quad s \in\left[0, s_{0}\right), \quad g_{0}=\mathrm{id},
$$

where $\xi:\left[0, s_{0}\right) \rightarrow \mathbb{R}$. See [20] (a derivation of this equation can be found in [13]). Our Theorems 1.1 and 1.2 remain true in this situation, and the proofs simplify slightly. There is one significant difference between (1.5) and (1.6). This is apparent by observing that the condition $\xi \in \operatorname{Lip}\left(\frac{1}{2}\right)$ for maps into $\mathbb{T}$, resp. $\mathbb{R}$, gives the same local regularity, but not global: the lift of $\xi: \mathbb{R} \rightarrow \mathbb{T}$ to $\mathbb{R}$ is not necessarily in $\operatorname{Lip}\left(\frac{1}{2}\right)$ if $\xi$ is. See the comment after the statement of Lemma 2.7 .

There are many examples of functions $\xi(s)$ which are not $\operatorname{Lip}\left(\frac{1}{2}\right)$ but which generate simple arcs. For instance, it is not hard to see that a disc slit by a smooth arc that meets $\mathbb{T}$ tangentially cannot have $\xi \in \operatorname{Lip}\left(\frac{1}{2}\right)$. A more interesting class of examples is $S L E_{\kappa}$ : if $0 \leq \kappa \leq 4$, then (1.6) with $\xi(t)=B(\kappa t)$ generates a simple arc in $\mathbb{H}$ almost surely, where $B$ is Brownian motion on $\mathbb{R}$. The same is true for the disc versions (1.1)-(1.5), with $\lambda(t)=e^{i B(\kappa t)}$. This is proved in 19. Previously O. Schramm 20] has proved that for $\kappa>4$, the Loewner equation almost never generates arcs in $\mathbb{H}$ (the boundary of $g_{s}(\mathbb{D})$ is still locally connected and therefore a curve, see [19, but these curves are not simple curves in $\mathbb{H}$ ).

For a slit domain with analytic slit, it is known that the driving term $\xi$ in the Loewner equation is real analytic. Recently, C. Earle and A. Epstein [4] have given a new proof of this fact and have also shown that for slits which are $C^{n}, \xi$ is at least $C^{n-1}$. Using the methods of our paper, one can obtain yet another proof for 
analytic arcs, and also show that $C^{n}$ curves yield $\xi$ in $C^{n-1 / 2}$. Details of this will appear elsewhere.

The Loewner equation is related to a certain algorithm for finding the conformal map onto a given simply connected domain, discovered independently by R. Kühnau [10] and the first author. This algorithm has been implemented (see http: \\www.math.washington.edu $\backslash \sim$ marshall) and converges extremely well in practice, even for conformal maps onto snowflake-like domains. In a forthcoming paper [16], we will prove convergence of this algorithm.

The paper is organized as follows: Section 2 contains a discussion of quasislitdiscs. In Section 3, we consider the Loewner equation on $\mathbb{T}$ and show quasisymmetry of the welding for a small $\operatorname{Lip}\left(\frac{1}{2}\right)$-norm. The main theorems are proved in Section 4. In Section 5, a pathological example is constructed.

Acknowledgement. We would like to thank Oded Schramm for numerous inspiring discussions. We would also like to thank the referee for a careful reading of the manuscript.

Update. After this paper was submitted, Joan Lind [12 proved that the unspecified constant $C$ in Theorem 1.1 is $C=4$, and that this is best possible.

\section{SLIT DISCS}

In this section we introduce the concept of quasislit-discs and establish their basic properties as needed in the proof of the main theorem.

Let $\Gamma \subset \overline{\mathbb{D}}$ be a Jordan arc with endpoints $a$ and $b$. We are only interested in arcs that begin in the circle $\mathbb{T}$ and are contained in $\mathbb{D} \backslash\{0\}, \Gamma \cap \mathbb{T}=\{a\}$, say. Call such a domain $\mathbb{D} \backslash \Gamma$ a slit disc with initial point $a$ and tip $b$. For every $0<t<1$ the domain $D_{t}=\mathbb{D} \backslash[t, 1]$ is a slit disc.

A slit disc $D$ is called a $K$-quasislit-disc if there is a $K$-quasiconformal homeomorphism $f$ of the sphere and $0<t<1$ such that $f\left(D_{t}\right)=D$ and $f(0)=0$. See the usual references [1] and [15] for background on quasiconformal maps, and [8], 18] for geometric properties of quasicircles (= images of circles or lines under quasiconformal maps). The requirement $f\left(D_{t}\right)=D$ implies $f(\mathbb{D})=\mathbb{D}$, and since quasiconformal homeomorphisms of the disc extend to the plane by reflection (with the same maximal dilatation $K$ ), it suffices to consider a $K$-quasiconformal homeomorphism $f$ of the disc.

Denote by $Q S D(K)$, resp. $Q S D(K, r)$, the set of all $K$-quasislit-discs, resp. those with conformal radius $\left|g^{\prime}(0)\right|=r$, where $g$ is a conformal map from $\mathbb{D}$ with $g(0)=0$. By the compactness of the family of $K$-quasiconformal maps fixing $\mathbb{D}$ and 0 we immediately obtain

Lemma 2.1. Equipped with the Carathéodory topology of domain convergence, $Q S D(K, r)$ is compact.

It is easy to see that the Carathéodory topology coincides with the topology induced by the Hausdorff distance $d\left(\partial D_{1}, \partial D_{2}\right)$ of the boundaries of domains of $Q S D(K, r)$. This is not true in the larger space of all slit discs.

We now give an analytic characterization in terms of the conformal welding. The conformal welding on a slit disc $D$ is defined as follows. Let $g: \mathbb{D} \rightarrow D$ be conformal with $g(0)=0$ and $g(1)=b$. Then there are two preimages $\alpha^{+}, \alpha^{-} \in \mathbb{T}$ of the initial point $a$. Let $I^{+}$be the oriented $\operatorname{arc}\left\langle 1, \alpha^{+}\right\rangle$and $I^{+}$-the $\operatorname{arc}\left\langle\alpha^{-}, 1>\right.$ on $\mathbb{T}$ and set $I=I^{+} \cup I^{-}$. Then the welding homeomorphism $\phi: I \rightarrow I$ is the unique continuous 
function with $\phi\left(\alpha^{+}\right)=\alpha^{-}$and

$$
g(x)=g(\phi(x)) \quad \text { for all } x \in I .
$$

Notice that $\phi(1)=1$.

Lemma 2.2. A slit disc $\mathbb{D} \backslash \Gamma$ is a quasislit-disc if and only if there is a constant $1 \leq M<\infty$ such that

$$
M^{-1} \leq \frac{|\phi(x)-1|}{|x-1|} \leq M
$$

for all $x \in I^{+}$and

$$
M^{-1} \leq \frac{|\phi(x)-\phi(y)|}{|\phi(y)-\phi(z)|} \leq M
$$

whenever $x, y, z \in I^{+}$(in this order) with $|x-y|=|y-z|$. Furthermore, the quasislit constant $K$ of $\mathbb{D} \backslash \Gamma$ depends on $M$ only, with $K \rightarrow 1$ if and only if $M \rightarrow 1$.

Proof. Suppose (2.1) and (2.2) hold. Let $T: \overline{\mathbb{D}} \rightarrow \overline{\mathbb{D}}$ be a quasiconformal map with $T(i)=\alpha^{+}, T(-i)=\alpha^{-}, T(1)=1$ and $T^{-1}(0) \in(-1,1)$. For instance, $T$ can be constructed as a composition of a quasiconformal map that sends $\alpha^{+}$and $\alpha^{-}$to symmetric points, followed by a Möbius transformation. It is easy to verify that $\varphi=T^{-1} \circ \phi \circ T$ satisfies (2.1) and (2.2) on $\langle 1, i\rangle$ and $\langle-i, 1\rangle$ (with a possibly larger constant $\left.M^{\prime}\right)$. We extend $\varphi$ to $\mathbb{T}$ by reflection in $i \mathbb{R}, \varphi(x)=\iota \varphi(\iota x)$, where $\iota x=-\bar{x}$. Next, define $\psi: \mathbb{T} \rightarrow \mathbb{T}$ by $\psi(x)=x$ on the upper half-circle $\langle 1,-1\rangle$, and $\psi(x)=\varphi(\bar{x})$ on $\langle-1,1\rangle$. It is not hard to check that $\psi$ is an orientation-preserving quasisymmetric homeomorphism, i.e. (2.2) holds for $\psi$ (with a constant $M^{\prime \prime}$ ) and all points $x, y, z$ on $\mathbb{T}$ with $|x-y|=|y-z|$. Furthermore $M^{\prime \prime}$ is close to 1 if $M$ is close to 1 . Since $\psi$ is quasisymmetric, it has a quasiconformal extension (again denoted by $\psi)$ to $\mathbb{D}$, and we can choose this extension such that $\psi([-1,1])=[-1,1]$. It follows that $\beta \equiv \psi^{-1}\left(T^{-1}(0)\right) \in(-1,1)$. Hence there is a unique $0<t<1$ such that a conformal map $h$ from $\mathbb{D}$ to $D_{t}$ exists with $h(\beta)=0, h(i)=h(-i)=1$ and $h(1)=t$. Indeed, $h$ is the composition of the maps

$$
\frac{1-z}{1+z}, c \sqrt{z^{2}+1} \text { and } \frac{1-z}{1+z}
$$

where $c=\left(\left(\frac{1-\beta}{1+\beta}\right)^{2}+1\right)^{-\frac{1}{2}}$ and $t=\frac{1-c}{1+c}$. Set $f=g \circ T \circ \psi \circ h^{-1}$. Then $f$ is a $K$-quasiconformal homeomorphism from $D_{t}$ onto $\mathbb{D} \backslash \Gamma$ that extends to a homeomorphism of $\mathbb{D}$. Since the interval $[t, 1]$ is removable for quasiconformal maps, $\mathbb{D} \backslash \Gamma$ is a quasislit-disc.

Conversely, if $D$ is a quasislit-disc and $f\left(D_{t}\right)=D$, consider the conformal maps $h: \mathbb{D} \rightarrow D_{t}$ with $h(0)=0, h(1)=t$ and $g: \mathbb{D} \rightarrow D$ with $g(0)=0, g(1)=b$ (the tip of $D)$. Then $\psi=g^{-1} \circ f \circ h$ is a $K$-quasiconformal self-map of the disc fixing 0 and 1 , and therefore extends quasisymmetrically to $\mathbb{T}$. Since $\phi(x)=\psi\left(\overline{\psi^{-1}(x)}\right)$ on $I$, the lemma follows.

Notice that the slit of a $K$-quasislit-disc obviously is a $K$-quasiarc (i.e., the image of a line segment under some $K$-quasiconformal map), whereas the converse is not true. Here is a geometric characterization.

Lemma 2.3. The slit disc $\mathbb{D} \backslash \Gamma$ is a quasislit-disc if and only if $\Gamma$ is a quasiarc that approaches $\mathbb{T}$ nontangentially. 
Proof. If $\mathbb{D} \backslash \Gamma$ is a quasislit-disc, then $\Gamma$ is a quasiarc by definition, and the nontangential approach follows from the quasi-invariance of the hyperbolic distance.

Conversely, a disc slit by a nontangential quasiarc is a John domain (see [18, Chapter 5 for the definition), with constant depending on the quasiarc constant and the nontangential approach only. It follows from the doubling property of harmonic measure in John domains that (2.1) and (2.2) hold. From Lemma 2.2 we obtain that $\mathbb{D} \backslash \Gamma$ is a quasislit-disc.

Remark. The proof shows that Lemma 2.3 is quantitative in the following sense: if $\Gamma$ is contained in the sector $\left\{z:-\alpha<\arg (1-\bar{a} z)<\alpha\right.$ and $\left.|a-z|<\frac{1}{2}\right\}$, then the quasislit constant $K$ of $\mathbb{D} \backslash \Gamma$ depends on $\alpha<\frac{\pi}{2}$ and the quasiarc constant $L$ of $\Gamma$ only. One can also show that $K \rightarrow 1$ as $\alpha \rightarrow 0$ and $L \rightarrow 1$. In the other direction, it is easy to see that $\alpha \rightarrow 0$ as $K \rightarrow 1$.

Lemma 2.4. Let $D=\mathbb{D} \backslash \Gamma$ be a $K$-quasislit-disc with initial point $a$ and tip $b$. Let $c$ and $d$ be points on $\Gamma$ such that the arc $\Gamma_{c}$ of $\Gamma$ from $c$ to a contains $\Gamma_{d}$. Let $\phi_{c}, \phi_{d}$ be conformal maps of $\mathbb{D}$ onto $\mathbb{D} \backslash \Gamma_{c}, \mathbb{D} \backslash \Gamma_{d}$ fixing 0 . Then $\phi_{d}^{-1}\left(\phi_{c}(\mathbb{D})\right)$ is a $K$-quasislit-disc, too.

Proof. There exists $0<t<1$ and a $K$-quasiconformal self-map $f$ of $\mathbb{D}$ fixing 0 such that $f\left(D_{t}\right)=D$. Set $t_{c}=f^{-1}(c)$ and $t_{d}=f^{-1}(d)$ so that $t \leq t_{c}<t_{d} \leq 1$. Let $h: \mathbb{D} \rightarrow D_{t_{d}}$ be conformal with $h(0)=0$ and $h^{\prime}(0)>0$. Then $F=\phi_{d}^{-1} \circ f \circ h$ is a $K$ quasiconformal homeomorphism of $\mathbb{D}$ fixing 0 with $F\left(D_{h^{-1}\left(t_{c}\right)}\right)=\phi_{d}^{-1}\left(\phi_{c}(\mathbb{D})\right)$.

Remark. Except for the sharp bound of the quasislit constant, Lemma 2.4 can also be obtained from the subinvariance principle of $[6]$.

Lemma 2.5. Let $D=\mathbb{D} \backslash \Gamma$ be a $K$-quasislit-disc with initial point a and tip $b$. Let $f: \mathbb{D} \rightarrow D$ be the conformal map with $f(0)=0$ and assume that $r:=f^{\prime}(0)>1 / 2$. Then

$$
\left|f^{-1}(b)-a\right| \leq C \sqrt{1-r}
$$

where $C$ depends continuously on $K$ with $C(1)=0$.

Proof. Set $A=f^{-1}(\Gamma)$. We are first going to show that

$$
\operatorname{diam} A \asymp \operatorname{diam} \Gamma \asymp \sqrt{1-r} \text {. }
$$

Let $\Gamma^{*}$ denote the reflection of $\Gamma$ in $\mathbb{T}$ and set $B=\Gamma \cup \Gamma^{*}$. Extend $f$ by reflection in $\mathbb{T}$ to a conformal map $f: \hat{\mathbb{C}} \backslash A \rightarrow \hat{\mathbb{C}} \backslash B$. With the usual notation $\Delta=\hat{\mathbb{C}} \backslash \overline{\mathbb{D}}$, consider the conformal maps $\phi: \Delta \rightarrow \hat{\mathbb{C}} \backslash A$ and $\psi: \Delta \rightarrow \hat{\mathbb{C}} \backslash B$ with positive derivative at $\infty$. The derivatives are cap $A$ and cap $B=(1 / r)$ cap $A$, where cap denotes logarithmic capacity ([7], page 74). Since cap $X \asymp \operatorname{diam} X$ for connected sets $X$, we obtain

$$
\operatorname{diam} A \asymp \operatorname{cap} A=\operatorname{rcap} B \asymp \operatorname{diam} B \asymp \operatorname{diam} \Gamma \text {. }
$$

Note also that diam $\Gamma \asymp 1-\operatorname{dist}(0, \Gamma)$ by Lemma 2.3. Since $\Gamma$ is a Jordan arc, $f$ is continuous on $\overline{\mathbb{D}}$ by the Continuity Theorem ([18, page 18). Moreover $|f|=1$ on $\partial \mathbb{D} \backslash A$ and thus

$$
\log \frac{1}{f^{\prime}(0)}=\int_{A} \log \left|\frac{e^{i \theta}}{f\left(e^{i \theta}\right)}\right| \frac{d \theta}{2 \pi} \leq \frac{|A|}{2 \pi} \log \frac{1}{\operatorname{dist}(0, \Gamma)},
$$


and we obtain

$$
1-r \leq C \frac{|A|}{2 \pi} \operatorname{diam} \Gamma \asymp(\operatorname{diam} A)^{2} .
$$

It remains to prove diam $A \leq C \sqrt{1-r}$. To this end, let $A^{\prime}=f^{-1}\left(B\left(b, \frac{1-|b|}{2}\right)\right)$. Then $|f| \leq(1+|b|) / 2$ on $A^{\prime}$. Since $1-|b| \asymp \operatorname{diam} \Gamma$, we obtain

$$
\log \frac{1}{f^{\prime}(0)} \geq \frac{\left|A^{\prime}\right|}{2 \pi} \log \frac{2}{1+|b|} \asymp\left|A^{\prime}\right|(1-|b|) \asymp\left|A^{\prime}\right| \operatorname{diam} \Gamma .
$$

The doubling property of harmonic measure in John domains implies $\left|A^{\prime}\right| \asymp|A|$, and we conclude that diam $A \leq C \sqrt{1-r}$, finishing the proof of (2.4).

Since $\psi=f \circ \phi$ and $f(0)=0$, we have

$$
z_{0}:=\phi^{-1}(0)=\psi^{-1}(0) \text {. }
$$

Now $\tilde{\phi}=\left(\phi-f^{-1}(b)\right) / \operatorname{cap} A$ and $\tilde{\psi}=(\psi-a) /$ cap $B$ are normalized univalent maps in $\Delta$ that omit the value 0 . Since diam $\hat{\mathbb{C}} \backslash g(\Delta) \leq 4$ for normalized univalent maps $g$, the functions $\tilde{\phi}(z)-z$ and $\tilde{\psi}(z)-z$ are analytic in $\Delta$ with boundary values of absolute value bounded above by 5 . The maximum principle yields

$$
\left|z_{0}+\frac{f^{-1}(b)}{\operatorname{cap} A}\right| \leq 5 \quad \text { and } \quad\left|z_{0}+\frac{a}{\operatorname{cap} B}\right| \leq 5
$$

Hence

$$
\left|\frac{f^{-1}(b)}{\operatorname{cap} A}-\frac{a}{\operatorname{cap} B}\right| \leq 10,
$$

and (2.3) follows since $\left|f^{-1}(b)\right|=|a|=1$.

The claim $C(1)=0$ can be proved by a normal families argument: let $D_{n}=$ $\mathbb{D} \backslash \Gamma_{n}$ be a sequence of $K_{n}$-quasislit-discs and $K_{n} \rightarrow 1$. We may assume that $a_{n}=1$. Then the $A_{n}$ (resp. $B_{n}$ ) "converge" (after rescaling) to a horizontal (resp. vertical) line segment. Therefore $\tilde{\psi}_{n}(z) \rightarrow z+1 / z$ and $\tilde{\phi}_{n}(z) \rightarrow z-1 / z$. Since $z_{n}=\phi_{n}^{-1}(0)=$ $\psi_{n}^{-1}(0) \rightarrow \infty$, it follows that $\tilde{\psi}_{n}\left(z_{n}\right)-\tilde{\phi}_{n}\left(z_{n}\right) \rightarrow 0$ and $\left|f_{n}^{-1}\left(b_{n}\right)-a_{n}\right|=o\left(\operatorname{cap} A_{n}\right)$ follows easily.

The proof of the Hölder continuity of the Loewner parameter for quasislit-discs is an immediate consequence of Lemmas 2.4 and 2.5] and is explained in Section 4. For the converse, we need to construct quasislit-discs with prescribed $a, f^{-1}(b)$ and $f^{\prime}(0)$. That this is possible is the content of the next lemma.

Lemma 2.6. Given $a, a^{\prime} \in \mathbb{T}$ and $0<r<1$, there is a slit disc $D=D_{a, a^{\prime}, r}$ with initial point $a$ and tip $b$ such that the conformal map $f: \mathbb{D} \rightarrow D$ with $f(0)=0$ satisfies

$$
f^{\prime}(0)=r \quad \text { and } \quad f\left(a^{\prime}\right)=b .
$$

Moreover, for $r \geq 1 / 2, D_{a, a^{\prime}, r}$ can be chosen as a $K$-quasislit-disc with

$$
K \leq C\left(\frac{\left|f^{-1}(b)-a\right|}{\sqrt{1-r}}\right)
$$

where $C:[0, \infty) \rightarrow[1, \infty)$ is continuous with $C(0)=1$.

Proof. Replacing $f(z)$ by $a f\left(a^{\prime} z\right)$ we see that it suffices to construct for each $w \in$ $\mathbb{D} \backslash\{0\}$ a conformal map $f$ to a slit disc with initial point 1 such that 1 maps to the tip and $f(0)=0, f^{\prime}(0)=w$ hold. 


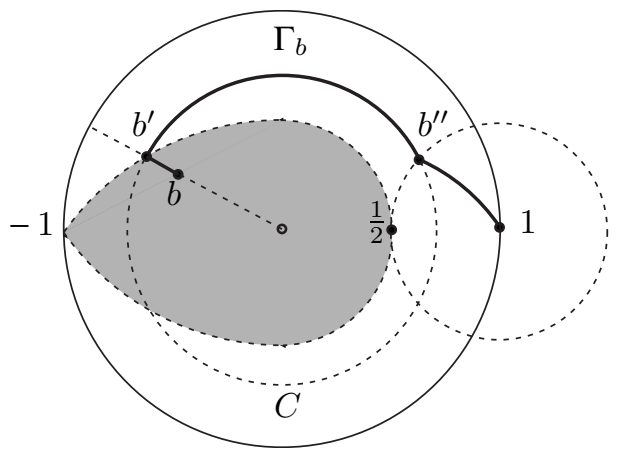

Figure 2.1. The region $d<1-|b|$ and a slit $\Gamma_{b}$

Define the canonical slits $S_{b}$ from points $b \in \mathbb{D} \backslash[-1,0]$ to 1 as follows: denoting by $T(z)=(1-z) /(1+z)$ the conformal map from $\mathbb{D}$ to the right half-plane, then

$$
S_{b}=T^{-1}[0, T(b)]
$$

Construct a family $D_{b}=\mathbb{D} \backslash \Gamma_{b}$ of slit discs with endpoint $b \in \mathbb{D} \backslash\{0\}$, together with the conformal maps $f_{b}$ from $\mathbb{D}$ to $D_{b}$ normalized by $f_{b}(0)=0, f_{b}(1)=b$, with the following properties:

(1) The function $m(b)=f_{b}^{\prime}(0)$ has a continuous extension to $\overline{\mathbb{D}}$, with $m(0)=0$ and $\left.m\right|_{\mathbb{T}}=$ identity.

(2) For $b \notin[-1,0], \Gamma_{b}$ has initial point 1 .

(3) For $|b-1|<1 / 2, \Gamma_{b}=S_{b}$.

A particular family with these properties is constructed as follows (see Figure 2.1). For $|b-1|<1 / 2$ we have to set $\Gamma_{b}=S_{b}$. For $|b-1| \geq 1 / 2$ but $b \notin[-1,0]$, let $d$ be the distance from $b$ to $[-1,0]$. If $d<1-|b|$, set $b^{\prime}=b /(d+|b|)$ and let $\Gamma_{b}$ be composed of the line segment $\left[b, b^{\prime}\right]$, the shorter arc of the circle $C=\left\{|z|=\left|b^{\prime}\right|\right\}$ from $b^{\prime}$ to the intersection $b^{\prime \prime}$ of $C$ with the circle $\{|z-1|=1 / 2\}$, and the canonical slit from $S_{b^{\prime \prime}}$. If $d \geq 1-|b|, \Gamma_{b}$ is composed of two arcs only, namely the circular arc of $C=\{|z|=|b|\}$ from $b$ to the intersection $b^{\prime \prime}$ of $C$ with the circle $\{|z-1|=1 / 2\}$, and $S_{b^{\prime \prime}}$. Finally set $\Gamma_{b}=[-1, b]$ for $b \in[-1,0]$. Then (2) and (3) are trivially satisfied, and (1) is an exercise in Carathéodory kernel convergence.

By topology, $m: \mathbb{D} \rightarrow \mathbb{D}$ is surjective and the existence claim of the lemma follows for $w \notin[-1,0]$. For the general case, repeat the above construction with an exceptional line segment $[0, i]$ instead of $[-1,0]$, say.

The estimate of the lemma follows from the fact that $\frac{\left|f^{-1}(b)-a\right|}{\sqrt{1-r}}$ is small only if $|b-1|<1 / 2$, and that the ratio is close to zero if and only if $b$ is close to $[1 / 2,1]$.

Finally we will prove a partial converse of Lemma 2.4. This is useful for reducing the proof of Theorem 1.2 to a bounded time interval $\left(s_{0}=1\right.$, say). If $D_{i}, 1 \leq i \leq n$, are slit discs with initial points $a_{i}$ and tips $b_{i}$ define the conformal concatenation

$$
D_{1} * D_{2} * \ldots * D_{n}=g_{n} \circ \ldots \circ g_{2} \circ g_{1}(\mathbb{D}),
$$

where $g_{i}: \mathbb{D} \rightarrow D_{i}$ are conformal maps such that $g_{i}\left(a_{i-1}\right)=b_{i}$ and $g_{i}(0)=0$. It is a slit disc too. The concatenation of quasislit-discs is a quasislit-disc, with constant depending on the constants of $D_{i}$ and on $n$. The next lemma says that if the 
conformal radii $\left|g_{i}^{\prime}(0)\right|$ are bounded away from 1 , the constant of the concatenation can be bounded independently of $n$.

Lemma 2.7. If $D_{i}$ are $K$-quasislit-discs and $\left|g_{i}^{\prime}(0)\right| \leq 1-\varepsilon$ for all $1 \leq i \leq n$, then $D_{1} * \ldots * D_{n}$ is a $K^{\prime}$-quasislit-disc with $K^{\prime}$ depending on $\varepsilon$ and $K$ only, and $K^{\prime} \rightarrow 1$ as $K \rightarrow 1$ with $\varepsilon$ fixed.

It is worthwhile to mention that the corresponding statement is not true for quasislit-planes if concatenation is defined using conformal maps with the hydrodynamic normalization instead of normalization in an interior point. This explains a difference between the "radial Loewner equation" (1.3) and the "chordal Loewner equation" (1.6) mentioned in the introduction: whereas the function $\lambda(t)=e^{i t}$ produces quasislit-discs with bounded $K$ (a logarithmic spiral), its lift $\xi(t)=t$ to $\mathbb{R}$ does not. The reason for this difference is that 0 is a hyperbolic (attractive) fixpoint for $g_{i}$, whereas $\infty$ is parabolic for conformal maps of $\mathbb{H}$ with hydrodynamic normalization.

Proof. We may assume that $\varepsilon$ is sufficiently small and all $D_{i}$ have conformal radius $\in[1-2 \varepsilon, 1-\varepsilon]$ (write $g_{i}$ as a composition of quasislit-maps, using Lemma 2.4). Let $\Gamma=\mathbb{D} \backslash\left(D_{1} * D_{2} * \ldots * D_{n}\right)$. Set $\gamma_{j}=\mathbb{D} \backslash D_{j}, \Gamma_{j}=g_{n} \circ \ldots \circ g_{j+1}\left(\gamma_{j}\right)$ and $\Gamma_{n}=\gamma_{n}$ so that $\Gamma=\bigcup_{j=1}^{n} \Gamma_{j}$. There are $r_{0}<1, r_{1}<1$ (depending on $\varepsilon$ on $K$ ) such that $g_{j+1}\left(\gamma_{j}\right) \subset D\left(0, r_{0}\right)$ and $\left|g_{j}(z)\right| \leq r_{1}|z|$ on $D\left(0, r_{0}\right)$. Hence there is an integer $k_{0}$ such that

$$
\Gamma_{j} \subset B\left(0, \frac{1}{2} \operatorname{dist}\left(\Gamma_{j+k}, 0\right)\right)
$$

for all $k \geq k_{0}$ and all $j \leq n-k$. We may assume that $\varepsilon$ is small enough to guarantee

$$
\operatorname{diam} \Gamma_{j} \leq \frac{1}{4} \operatorname{dist}\left(\Gamma_{j}, 0\right) .
$$

To prove the lemma if $n$ is bounded is easy using compactness. Hence we may use the lemma for $k_{0}$ and assume $n>k_{0}$. Then

$$
\Gamma_{j} \cup \ldots \cup \Gamma_{j+k_{0}-1} \text { is a quasiarc for all } j
$$

with constant depending on $K$ and $\varepsilon$. To see this, first apply the lemma to $D_{j} *$ $D_{j+1} * \ldots * D_{j+k_{0}-1}$ to obtain a quasislit-disc, then apply $g_{j+k_{0}-1}$ which changes the constant only by a bounded amount, and finally apply $g_{n} \circ \ldots \circ g_{j+k_{0}}$, using the elementary fact that every conformal map $g$ in $\mathbb{D}$ has a $K\left(r_{0}\right)$-qc extension from $D\left(0, r_{0}\right)$ to $\mathbb{C}$. An explicit bound $K\left(r_{0}\right) \leq\left(1+r_{0}\right) /\left(1-r_{0}\right)$ follows from the $\lambda$ Lemma (see [18, Chapter 5.6) applied to the holomorphic motion $(z, \lambda) \mapsto g(\lambda z) / \lambda$ and setting $\lambda=r_{0}$.

It is routine to check that every arc $\Gamma$ satisfying (2.5), (2.6) and (2.7) also satisfies the Ahlfors three point property, hence is a quasiarc. It also follows that the set $\Gamma \cup\{0\}$ has the three point property, and from (2.7) it follows that $\Gamma$ meets $\mathbb{T}$ nontangentially. This implies that $\mathbb{D} \backslash \Gamma$ is a $K^{\prime}$-quasislit-disc.

To prove that $K^{\prime} \rightarrow 1$ as $K \rightarrow 1$ is easy (using compactness) for each fixed $n$. To prove independence of $n$ goes along the same lines as the above proof. We omit the details. 


\section{The Loewner equation And CONFORMal Welding}

Let $\xi:\left[0, s_{0}\right] \rightarrow \mathbb{T}$ be continuous and let $g_{s}$ be the solution to (1.5),

$$
\partial_{s} g_{s}(z)=-g_{s}(z) \frac{\xi(s)+g_{s}(z)}{\xi(s)-g_{s}(z)}, \quad g_{0}(z)=z .
$$

There is no loss of generality in assuming $\xi(0)=1$. For ease of notation, extend $\xi$ to $[0, \infty)$ by $\xi(s) \equiv \xi\left(s_{0}\right)$ for $s>s_{0}$. Notice that $g_{s}^{\prime}(0)=e^{-s}$. First let us assume that $g_{s_{0}}(\mathbb{D})=\mathbb{D} \backslash \Gamma_{s_{0}}$ is a slit disc. Then $g_{s}(\mathbb{D})=\mathbb{D} \backslash \Gamma_{s}$ is a slit disc with initial point $\xi(s)$ for all $s \in\left[0, s_{0}\right]$. By Schwarz reflection, $g_{s}$ is a conformal map of $\hat{\mathbb{C}} \backslash g_{s}^{-1}\left(\Gamma_{s}\right)$ onto $\hat{\mathbb{C}} \backslash\left(\Gamma_{s} \cup \Gamma_{s}^{*}\right)$, where ${ }^{*}$ denotes reflection in $\mathbb{T}$. Furthermore, (3.1) holds throughout $\mathbb{C} \backslash g_{s}^{-1}\left(\Gamma_{s}\right)$. Roughly speaking, the slit $\Gamma_{s_{0}}$ is formed as follows. Points on $\mathbb{T}$ move along $\mathbb{T}$ towards the singularity $\xi(s)$ according to (3.1), until they actually meet the singularity. At this time, they leave the circle and move into $\mathbb{D}$. Points hit the singularity pairwise (from both sides of $\xi(s)$ ); hence points on $\mathbb{T}$ are identified under the welding homeomorphism if and only if they hit the singularity at the same time and before time $s_{0}$. More formally, for $z \in \mathbb{T}$ denote

$$
T(z)=\sup \left\{s>0: g_{t}(z) \neq \xi(t) \text { for all } t \in[0, s]\right\} .
$$

If $T(z)<\infty$ we have $g_{T(z)}(z)=\xi(T(z))$, and we could have defined $T(z)$ by setting $T(z)=s$ for both preimages $g_{s}^{-1}(\xi(s))$.

As in Section 2, set $I=g_{s_{0}}^{-1}\left(\Gamma_{s_{0}}\right) \subset \mathbb{T}$ and consider the welding $\phi: I \rightarrow I$ that interchanges the endpoints of $I$ and satisfies

$$
g_{s_{0}}(x)=g_{s_{0}}(\phi(x)) \quad \text { for all } x \in I ;
$$

in particular $\phi(1)=1$. For $z \in I$ we then have

$$
T(z)=T\left(z^{\prime}\right) \quad \text { if and only if } \quad \phi(z)=z^{\prime} \text { or } z=z^{\prime} .
$$

In the special case $\xi(t)=\xi_{0}(t) \equiv 1$, calculation shows

$$
g_{t}(z)=e^{t}\left(1+z\left(2-2 e^{-t}+z\right)-(1+z) \sqrt{1+z\left(2-4 e^{-t}+z\right)}\right) /(2 z)
$$

so that

$$
g_{t}^{-1}(1)=2 e^{-t}-1 \pm i 2 e^{-t} \sqrt{e^{t}-1}
$$

and

$$
T_{0}\left(e^{i \varphi}\right)=-\log \frac{1+\cos \varphi}{2}
$$

For later use, write

$$
G(t, x)=\arg g_{t}\left(e^{i x}\right) .
$$

Now let us drop the assumption of $g_{s_{0}}(\mathbb{D})$ being a slit disc. Then we can still define $T(z)$ by (3.2), and we would like to show that under the assumption of $\|\xi\|_{\frac{1}{2}}$ being small, pairs of points $z, z^{\prime}$ hit the singularity in finite time.

Lemma 3.1. There is a constant $C_{0}$ such that if $\|\xi\|_{\frac{1}{2}}<C_{0}$, then

$$
C^{-1} \leq \frac{T(z)}{T_{0}(z)} \leq C
$$

for all $z \in \mathbb{T}$ with $\Re z>0$, where $C=C\left(\|\xi\|_{\frac{1}{2}}\right) \rightarrow 1$ as $\|\xi\|_{\frac{1}{2}} \rightarrow 0$. 
Proof. The idea is quite simple and best explained for the half-plane version (1.6), $\partial_{s} g_{s}(x)=2 /\left(\xi(s)-g_{s}(x)\right)$ : if $\xi(0)=0, x>0$ and $s=\varepsilon x^{2}$ for some small fixed $\varepsilon$, then $|\xi(t)|<x \sqrt{\varepsilon}|| \xi \|_{\frac{1}{2}}$ for $t \leq s$ so that $g_{s}(x)-\xi(s) \sim x-2 s / x-\xi(s) \sim\left(1-C^{\prime}\right) x$, so that $g_{s}-\xi$ made a definite advance towards 0 . Repeating this argument one can estimate the time $T$ for which $g_{s}-\xi=0$. This idea can easily be made precise, but we need some notation.

Fix $z=e^{i \varphi_{0}}$ with $0<\varphi_{0} \leq \pi / 2$ and set $\varphi(s)=\arg g_{s}(z)=-i \log g_{s}(z)$, so $\varphi(0)=\varphi_{0}$. Writing $\xi(s)=e^{i \psi(s)}$ with $\psi(0)=0$ we have

$$
\varphi^{\prime}(s)=-i \frac{\partial_{s} g_{s}(z)}{g_{s}(z)}=i \frac{\xi(s)+g_{s}(z)}{\xi(s)-g_{s}(z)}=-\cot \frac{\varphi(s)-\psi(s)}{2}
$$

and in particular (the case $\psi \equiv 0$ )

$$
\partial_{s} G(s, \tau)=-\cot \frac{G(s, \tau)}{2} .
$$

Define for $0<\varphi_{1}<\varphi_{0}$,

$$
T\left(\varphi_{0}, \varphi_{1}\right)=\inf \left\{t: \varphi(t)-\psi(t)=\varphi_{1}\right\},
$$

and for $0<\varphi_{1}<\varphi_{2} \leq \pi / 2$,

$$
T_{0}\left(\varphi_{2}, \varphi_{1}\right)=\inf \left\{t: G\left(t, \varphi_{2}\right)=\varphi_{1}\right\}=-\log \frac{1+\cos \varphi_{2}}{2}+\log \frac{1+\cos \varphi_{1}}{2} .
$$

We claim that there is a universal constant $\varepsilon>0$ and $C=C\left(\|\xi\|_{\frac{1}{2}}\right)$ with $C(t) \rightarrow 1$ as $t \rightarrow 0$, such that

$$
\frac{1}{C} \leq \frac{T_{0}\left(\varphi_{0},(1-\varepsilon) \varphi_{0}\right)}{T\left(\varphi_{0},(1-\varepsilon) \varphi_{0}\right)} \leq C .
$$

Repeated application of this estimate and summation yields the lemma.

To prove the estimate, fix a small constant $\varepsilon$ and set

$$
\sigma:=T_{0}\left(\varphi_{0},(1-\varepsilon) \varphi_{0}\right) \leq C \varepsilon \varphi_{0}^{2},
$$

where we have used (3.4). Then $|\psi(s)| \leq \delta:=\sqrt{\sigma}\|\xi\|_{\frac{1}{2}}=o\left(\varphi_{0}\right)$ for $0 \leq s \leq \sigma$, where the $o\left(\varphi_{0}\right)$ refers to $\|\xi\|_{\frac{1}{2}} \rightarrow 0$. From (3.5) and (3.6) we get

$$
-\cot \frac{\varphi(s)-\delta}{2} \leq \varphi^{\prime}(s) \leq-\cot \frac{\varphi(s)+\delta}{2}, \quad \partial_{s} G\left(s, \varphi_{0} \pm \delta\right)=-\cot \frac{G\left(s, \varphi_{0} \pm \delta\right)}{2}
$$

and obtain

$$
G\left(s, \varphi_{0}-\delta\right)+\delta \leq \varphi(s) \leq G\left(s, \varphi_{0}+\delta\right)-\delta .
$$

Using $\left|\partial_{x} G(\sigma, x)\right|<C_{1}$ for $\sigma<C_{2} x^{2}$ we obtain

$\varphi(\sigma)-\psi(\sigma) \leq G\left(\sigma, \varphi_{0}+\delta\right) \leq G\left(\sigma, \varphi_{0}\right)+C_{1} \delta=(1-\varepsilon) \varphi_{0}+C_{1} \delta=(1-\varepsilon+o(1)) \varphi_{0}$ as $\|\xi\|_{\frac{1}{2}} \rightarrow 0$. The lower bound $\varphi(\sigma)-\psi(\sigma) \geq(1-\varepsilon-o(1)) \varphi_{0}$ is obtained in the same way, and (3.8) and the lemma follow easily.

Lemma 3.2. There is a constant $C_{0}$ such that if $\|\xi\|_{\frac{1}{2}}<C_{0}, z_{1}, z_{2}, z_{3} \in I^{+} \cap$ $\{\Re z>0\}$ and

then

$$
\left|z_{1}-z_{2}\right|=\left|z_{2}-z_{3}\right|,
$$

where $C \rightarrow 1$ as $\|\xi\|_{\frac{1}{2}} \rightarrow 0$.

$$
\frac{1}{C} \leq \frac{\left|\phi\left(z_{1}\right)-\phi\left(z_{2}\right)\right|}{\left|\phi\left(z_{2}\right)-\phi\left(z_{3}\right)\right|} \leq C
$$


Proof. Write $z_{j}=e^{i \varphi_{j}}, g_{s}\left(z_{j}\right)=e^{i \varphi_{j}(s)}$ and $\xi(t)=e^{i \psi(t)}$. After relabeling we may assume $0<\varphi_{1}<\varphi_{2}<\varphi_{3}$. If $\varphi_{3}-\varphi_{1} \geq \varphi_{1}$, then the lemma follows from Lemma 3.1 and (3.4) since $T\left(z_{j}\right)=T\left(\phi\left(z_{j}\right)\right)$ and since we may assume that $\|\xi\|_{\frac{1}{2}}$ is close to zero. Hence we may assume $\varphi_{3}-\varphi_{1}<\varphi_{1}$.

Since $\left(\varphi_{1}(t)-\psi(t)\right) /\left(\varphi_{3}(t)-\varphi_{1}(t)\right) \rightarrow 0$ as $t \rightarrow T\left(z_{1}\right)$, there is $T$ such that $\varphi_{1}(T)-\psi(T)=\varphi_{3}(T)-\varphi_{1}(T)$. Set

$$
q(t)=\frac{\varphi_{3}(t)-\varphi_{1}(t)}{\varphi_{2}(t)-\varphi_{1}(t)}
$$

We claim that $q(t)$ is bounded for $0 \leq t \leq T$. In fact, we will prove that $q(t) / q(0)$ is bounded for $0 \leq t \leq T$ whenever $0<\varphi_{1}<\varphi_{2}<\varphi_{3}$ and $\varphi_{3}-\varphi_{1}<\varphi_{1}$, without assuming that $\varphi_{3}-\varphi_{2}=\varphi_{2}-\varphi_{1}:$ set

$$
d_{j}(t)=\varphi_{j}(t)-\psi(t)>0 \quad \text { and } \quad \delta(t)=\varphi_{3}(t)-\varphi_{1}(t)>0
$$

From (3.5) and $d_{1}(t)<d_{3}(t) \leq 2 d_{1}(t)$ for $0 \leq t \leq T$ we have

$$
\partial_{t} \delta=\frac{\sin \left(\varphi_{3}-\varphi_{1}\right) / 2}{\sin \left(d_{3} / 2\right) \sin \left(d_{1} / 2\right)} \asymp \frac{\varphi_{3}-\varphi_{1}}{d_{1}^{2}}
$$

and

$$
\begin{aligned}
\partial_{t} \log q & =\frac{1}{\sin \left(d_{1} / 2\right)}\left(\frac{\sin \left(\varphi_{3}-\varphi_{1}\right) / 2}{\left(\varphi_{3}-\varphi_{1}\right) \sin \left(d_{3} / 2\right)}-\frac{\sin \left(\varphi_{2}-\varphi_{1}\right) / 2}{\left(\varphi_{2}-\varphi_{1}\right) \sin \left(d_{2} / 2\right)}\right) \\
& \asymp \frac{\varphi_{3}-\varphi_{2}}{d_{1} d_{2} d_{3}}+O(1),
\end{aligned}
$$

again using $d_{3}(t) \leq 2 d_{1}(t)$.

Since $\partial_{t} \delta>0$ we have $\varphi_{3}(t)-\varphi_{2}(t)<\delta(t)<\delta(T)=d_{1}(T)$, and we obtain

$$
\left|\partial_{t} \log q(t)\right| \leq C d_{1}(T) / d_{1}(t)^{3}+O(1) .
$$

From Lemma 3.1 and (3.4) we know that $T\left(e^{i \varphi}\right) \asymp \varphi^{2}$; hence $T \leq C$. Write $T(z)=$ $s+\tilde{T}_{s}\left(g_{s}(z)\right)$ with $\tilde{T}_{s}$ being the time where the image of $g_{s}(z)$ under the Loewner flow associated with the function $t \mapsto \xi(t+s)$ hits the singularity $\xi(t+s)$. Lemma 3.1 yields $\tilde{T}_{s}\left(e^{i \varphi}\right) \asymp(\varphi-\psi(s))^{2}$, and we obtain

$$
d_{1}(s)^{2} \asymp \tilde{T}_{s}\left(g_{s}\left(z_{1}\right)\right)=T\left(z_{1}\right)-s=T+\tilde{T}_{T}\left(g_{T}\left(z_{1}\right)\right)-s \geq T-s+C d_{1}(T)^{2} .
$$

Thus

$$
\begin{aligned}
\log q(T) / q(0) & \leq C \int_{0}^{T}\left(\frac{d_{1}(T)}{d_{1}(t)^{3}}+O(1)\right) d t \\
& \leq C d_{1}(T) \int_{C d_{1}(T)^{2}}^{\infty} s^{-\frac{3}{2}} d s+O(1)=O(1) .
\end{aligned}
$$

Similarly one can prove that $\left(\varphi_{3}(t)-\varphi_{1}(t)\right) /\left(\varphi_{3}(t)-\varphi_{2}(t)\right)$ is bounded.

To finish the proof of the lemma, we can argue as in the case $\varphi_{3}-\varphi_{1} \geq \varphi_{1}$ to obtain that $\left|\left(g_{T}\left(\phi\left(z_{1}\right)\right)-g_{T}\left(\phi\left(z_{2}\right)\right)\right) /\left(g_{T}\left(\phi\left(z_{2}\right)\right)-g_{T}\left(\phi\left(z_{3}\right)\right)\right)\right|$ is bounded above and below, and then use the boundedness of the quotient (now running the equation backwards from time $T$ to 0$)$ to conclude that $\left|\left(\phi\left(z_{1}\right)-\phi\left(z_{2}\right)\right) /\left(\phi\left(z_{2}\right)-\phi\left(z_{3}\right)\right)\right|$ is bounded above and below. 


\section{Proof of the theorems}

In the introduction we have described how to pass from a Loewner chain $f_{t}$ associated with a slit domain to the equation (1.5). This can be reversed: If $g_{s}$ solves (1.5), $0 \leq s \leq s_{0}$, then

$$
f_{t}=g_{s_{0}} \circ g_{s_{0}+t}^{-1}, \quad-s_{0} \leq t \leq 0
$$

is a Loewner chain (more precisely, $f_{t}$ has an analytic extension to $\mathbb{D}$ that constitutes a Loewner chain) satisfying (1.3), and $f_{-s_{0}}=g_{s_{0}}, f_{0}=i d$, and $\lambda(t)=\xi\left(s_{0}+t\right)$. This follows from observing that all $f_{t}$ are well-defined on some subdomain of $\mathbb{D}$, that (1.3) holds there because of (1.5), and that (1.3) has analytic solutions in all of $\mathbb{D}$. See $([17$, Theorem 6.2$)$. Set

$$
\phi_{s, t}=f_{s}^{-1} \circ f_{t} .
$$

For $-s_{0}=t_{0}<t_{1}<\ldots<t_{n}=0$ we have

$$
g_{s_{0}}=f_{-s_{0}}=\phi^{(n)} \circ \phi^{(n-1)} \circ \ldots \circ \phi^{(1)}, \quad \phi^{(j)}=\phi_{t_{j}, t_{j-1}} .
$$

The $\phi^{(j)}$ are the (unique) solutions to

$$
\partial_{s} \phi_{s}^{(j)}(z)=-\phi_{s}^{(j)}(z) \frac{\lambda_{j}(s)+\phi_{s}^{(j)}(z)}{\lambda_{j}(s)-\phi_{s}^{(j)}(z)}, \quad \lambda_{j}(s)=\lambda\left(t_{j-1}+s\right), \quad \phi_{0}^{(j)}=i d
$$

with $0 \leq s \leq t_{j}-t_{j-1}$. Conversely, if solutions $\phi^{(j)}$ to (4.2) are given with $\lambda_{j}\left(t_{j}-\right.$ $\left.t_{j-1}\right)=\lambda_{j-1}(0)$, then the solutions $f_{-s_{0}}$ resp. $g_{s_{0}}$ to (1.3) resp. (1.5) are just $\phi^{(n)} \circ \phi^{(n-1)} \circ \ldots \circ \phi^{(1)}$. In particular, if all $\phi^{(j)}$ map to slit discs, then $f_{t}$ and $g_{s}$ map to slit discs.

Proof of Theorem 1.2 . Let us first assume that $g_{s_{0}}(\mathbb{D})=\mathbb{D} \backslash \Gamma$ is a $K$-quasislit-disc. Consider the Loewner chain (4.1) described above. By Lemma 2.4, $f=f_{s}^{-1} \circ f_{t}=$ $g_{s+s_{0}} \circ g_{t+s_{0}}^{-1}$ maps onto a $K$-quasislit-disc. Since the initial point of $f(\mathbb{D})$ is $a=\xi(s)$ and the tip is $b=f(\xi(t))$, we have

$$
|\xi(t)-\xi(s)| \leq C \sqrt{1-e^{t-s}} \leq C \sqrt{s-t}
$$

by Lemma 2.5. which proves $\xi \in \operatorname{Lip}\left(\frac{1}{2}\right)$.

Now let $\xi:\left[0, s_{0}\right) \rightarrow \mathbb{T}$ with small $\|\xi\|_{\frac{1}{2}}$ be given. We will first assume $s_{0} \leq \sigma_{0}$ where $\sigma_{0}$ is small enough to ensure that $T(z)>\sigma_{0}$ for $|z|=1, \Re z<0$ (see Lemma 3.1). In order to approximate $\xi$ by functions $\xi_{n} \in \operatorname{Lip}\left(\frac{1}{2}\right)$, set

$$
t_{j}=\frac{j}{n} s_{0}, \quad \xi_{j}=\xi\left(t_{j}\right), \quad r=e^{-\frac{s_{0}}{n}}
$$

for $0 \leq j \leq n$, and let

$$
D_{j}=D_{\xi_{j+1}, \xi_{j}, r}
$$

be the slit disc provided by Lemma 2.6. Thus the normalized conformal map $\phi^{(j)}: \mathbb{D} \rightarrow D_{j}$ maps $\xi_{j}$ to the tip of $D_{j}$, and $\xi_{j+1}$ is the base. Let $\lambda_{j}:\left[0, \frac{s_{0}}{n}\right] \rightarrow \mathbb{T}$ be the Loewner parameter of $D_{j}$ (i.e., the function obtained from (1.3) for $\Omega=D_{j}$ ). Then $\lambda_{j}(0)=\xi_{j}, \lambda_{j}\left(\frac{s_{0}}{n}\right)=\xi_{j+1}$ and $\left\|\lambda_{j}\right\|_{\frac{1}{2}} \leq C$, where $C=C\left(\|\xi\|_{\frac{1}{2}}\right)$ is a constant depending only on $\|\xi\|_{\frac{1}{2}}$. The latter follows from $D_{j}$ being a quasislit-disc by Lemma 2.6. combined with the already proven part of the theorem. Notice that $C(t) \rightarrow 0$ as $t \rightarrow 0$.

As explained above, the composition

$$
g_{s_{0}}^{(n)}=\phi^{(n-1)} \circ \ldots \circ \phi^{(0)}
$$


is the solution to (1.3) with parameter $\xi_{n}:\left[0, s_{0}\right] \rightarrow \mathbb{T}$ given by

$$
\left.\xi_{n}\right|_{\left[t_{j}, t_{j+1}\right]}(t)=\lambda_{j}\left(t-t_{j}\right) \text {. }
$$

It follows that

$$
\left\|\xi_{n}\right\|_{\frac{1}{2}} \leq 2 C\left(\|\xi\|_{\frac{1}{2}}\right)+\|\xi\|_{\frac{1}{2}}
$$

and the right-hand side is small if $\|\xi\|_{\frac{1}{2}}$ is small.

From Lemmas 2.2, 3.1 and 3.2 it follows that $D(n)=g_{s_{0}}^{(n)}(\mathbb{D})$ is a quasislit-disc. Indeed, by the assumption $s_{0} \leq \sigma_{0}$ we have $I^{+} \subset\{\operatorname{Re} z>0\}$. Hence (2.1) follows from Lemma 2.2. (3.4), and Lemma 3.1, whereas (2.2) follows from Lemma 3.2 . Finally, the case $s_{0}>\sigma_{0}$ follows from Lemma 2.7

By Lemma 2.1, $g_{s}^{(n)}(\mathbb{D})$ converges to a conformal map $g_{s}$ onto a quasislit-disc. Since $\xi_{n}$ converges to $\xi$, passing to the limit in (1.5) on $\mathbb{D}$, we conclude that $g_{s}$ must also satisfy (1.5) and Theorem 1.2 follows.

Proof of Theorem 1.1. If $\gamma$ is a quasiarc, then the proof of Lemma 2.4 shows that $\phi_{s, t}=f_{s}^{-1} \circ f_{t}$ maps $\mathbb{D}$ onto quasislit-discs, and $\lambda \in \operatorname{Lip}\left(\frac{1}{2}\right)$ follows from Lemma 2.5 .

Conversely, if $\|\lambda\|_{\frac{1}{2}}$ is small, then the solutions $g_{s}$ of (1.3) with $\xi(s)=\lambda(s)$ map to quasislits by Theorem 1.2. It is well known that $e^{s} g_{s} \rightarrow f_{0}$ locally uniformly in $\mathbb{D}$ as $s \rightarrow \infty$ (see [17, Theorem 6.3). By compactness of the space of quasislit-planes with conformal radius 1 , the theorem follows.

\section{EXAmples}

We want to construct a connected unbounded set that is not locally connected such that the driving term $\lambda(t)$ in the Loewner equation is in $\operatorname{Lip}\left(\frac{1}{2}\right)$. To this end, let $\gamma_{1}$ be a logarithmic spiral; for instance, $\gamma_{1}(t)=t e^{i \log |t|},-1 \leq t \leq 1$. Let $\gamma_{2}$ be a smooth curve joining one endpoint of $\gamma_{1}$ to $\infty$ within $\mathbb{C} \backslash \gamma_{1}$. Let $F: \mathbb{C} \backslash\{0\} \rightarrow \mathbb{C} \backslash \overline{\mathbb{D}}$ be the homeomorphism $F(z)=(|z|+1) z /|z|$. Then $\Gamma=F\left(\gamma_{1} \cup \gamma_{2}\right)$ has the desired properties: clearly $\Gamma$ is not locally connected. Fix a point $z_{0} \in \mathbb{C} \backslash(\Gamma \cup \mathbb{D})$. Then $\mathbb{C} \backslash \Gamma$ can be embedded in a Loewner chain (with respect to $z_{0}$ ) as follows: fix a parametrization $\gamma$ of $\gamma_{1} \cup \gamma_{2}$ and set $t_{0}=\gamma^{-1}(0)$. The domains $\hat{D}_{t}=\mathbb{C} \backslash \gamma[t, \infty)$ are continuously increasing. Set $D_{t}=F\left(\hat{D}_{t}\right)$ for $t \leq t_{0}$ and $D_{t}=F\left(\hat{D}_{t}\right) \cup \mathbb{D}$ for $t>t_{0}$. It is easy to check that for $s<t$, there is a crosscut $c_{s, t}$ of $D_{t}$ separating $z_{0}$ from $D_{t} \backslash D_{s}$ with diam $c_{s, t} \rightarrow 0$ as $s \rightarrow t$. Thus the condition of (13], Theorem $2.6)$ is satisfied and $D_{t}$ together with the conformal maps $f_{t}: \mathbb{D} \rightarrow D_{t}, f_{t}(0)=z_{0}$ form a Loewner chain satisfying (1.3) with continuous $\lambda$ (assuming that $\left(D_{t}\right)$ is parametrized such that $\left.f_{t}^{\prime}(0)=e^{t}\right)$. In order to show $\lambda \in \operatorname{Lip}\left(\frac{1}{2}\right)$, it suffices to show

$$
\left|\lambda(t)-\lambda\left(t_{0}\right)\right| \leq C\left|t-t_{0}\right|^{\frac{1}{2}}
$$

Assume $t<t_{0}$ (the proof for $t>t_{0}$ is similar). Define a sequence $T_{n} \nearrow t_{0}$ inductively by choosing $T_{0}<t_{0}$ and setting

$$
T_{n+1}=\inf \left\{t>T_{n}:\left|\Gamma(t)-\Gamma\left(T_{n}\right)\right|=\left|\Gamma\left(T_{n}\right)\right|-1\right\} .
$$

Since consecutive turns of the logarithmic spiral $\gamma_{1}$ have geometrically decreasing distances from 0 , we have that $\left|\Gamma\left(T_{n+1}\right)-\Gamma\left(T_{n}\right)\right|$ is comparable to the distance of $\Gamma\left(T_{n}\right)$ to the "previous turn" of $\Gamma$. Then it is easy to see that $\Gamma_{n}=\Gamma\left[T_{n}, T_{n+1}\right]$ has harmonic measure

$$
\omega_{n}=\omega\left(z_{0}, \Gamma_{n}, D_{T_{n}}\right)
$$




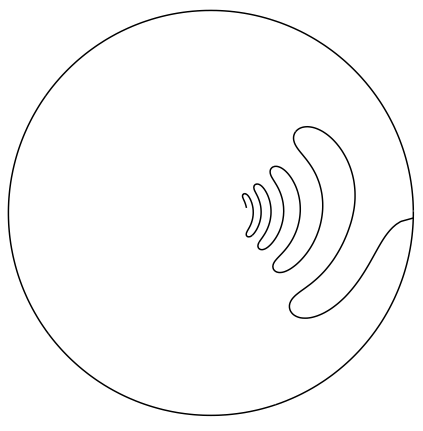

$\lambda(t)=\sin (8 \pi t)$

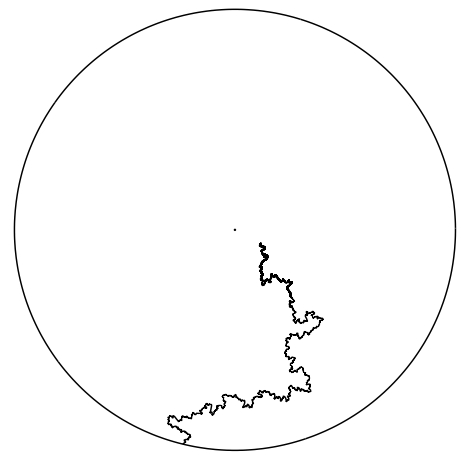

$\lambda(t)=$ random walk

FigURE 5.1.

satisfying

$$
\omega_{n+k} \leq C_{1} e^{-C_{2} k} \omega_{n} \quad \text { for all } \quad k \geq 1
$$

Furthermore, $f_{T_{n}}^{-1}\left(\Gamma_{n}\right)$ is a smooth quasislit (with uniformly bounded $K$ ). From (2.4) we obtain

$$
\left|\lambda\left(T_{n+1}\right)-\lambda\left(T_{n}\right)\right| \leq C \operatorname{diam} f_{T_{n}}^{-1}\left(\Gamma_{n}\right) \leq C \omega_{n}
$$

By Lemma 2.5.

$$
T_{n+1}-T_{n} \geq C \omega_{n}^{2}
$$

and the claim (5.1) follows from (5.2) by summation.

Finally we would like to mention that our proof of Theorem 1.2 leads to a simple algorithm for approximating the solutions of (1.3): The compositions (4.3) are converging to $g_{s}$ as proved above, so the problem reduces to finding the maps $\phi^{(j)}$. Using the conformal maps $z \mapsto(z+a)^{a}(z+1-a)^{1-a}$ of the upper half-plane to the half-plane slit by a line segment of angle $a \pi$, this problem essentially reduces to finding the exponents $a$. To do this numerically is not hard. This algorithm has been implemented by our undergraduate research assistants Tarn Adams, Gary Look and Julie Rowlett, whom we would like to thank for their effort. The two pictures in Figure 5.1 were produced with this algorithm. In the second picture, $\lambda(t)$ is the random walk

$$
\lambda\left(\frac{j}{n}\right)=\exp \left(i \sqrt{\frac{2}{n}} \sum_{k=0}^{j} \pm 1\right), \quad 0 \leq j \leq n .
$$

Since this approximates $B(2 t)$, this should (and does) look like a "typical" path of the Loop Erased Random Walk by [20].

\section{REFERENCES}

[1] L. Ahlfors, Lectures on quasiconformal mappings, Van Nostrand (1966). MR0200442 (34:336)

[2] L. de Branges, A proof of the Bieberbach conjecture, Acta Math. 154 (1985), 137-152. MR0772434 (86h:30026)

[3] L. Carleson, N. Makarov, Aggregation in the plane and Loewner's equation, Comm. Math. Phys. 216 (2001), 583-607. MR.1815718 (2001m:82079) 
[4] C. Earle, A. Epstein, Quasiconformal variation of slit domains, Proc. Amer. Math. Soc. 129 (2001), 3363-3372. MR.1845014 (2002f:30009)

[5] P. Duren, Univalent functions, Springer (1983). MR0708494 (85j:30034)

[6] J. L. Fernández, J. Heinonen, O. Martio, Quasilines and conformal mappings, J. Analyse Math. 52 (1989), 117-132. MR0981499(90a:30017)

[7] J. Garnett, D. E. Marshall, Harmonic Measure, Cambridge University Press (2005).

[8] F. Gehring, Characteristic properties of quasidisks, Presses de l'Université de Montréal, Montreal, 1982. MR0674294 (84a:30036)

[9] P. P. Kufarev, A remark on the integrals of the Loewner equation, Dokl. Akad. Nauk SSSR, 57 (1947), 655-656 (in Russian) MR0023907(9:421d)

[10] R. Kühnau, Numerische Realisierung konformer Abbildungen durch "Interpolation" Z. Angew. Math. Mech. 63 (1983), 631-637 (in German). MR0737000(85b:30012)

[11] K. Löwner, Untersuchungen über schlichte konforme Abbildungen des Einheitskreises, I, Math. Ann. 89 (1923), 103-121.

[12] J. Lind, A sharp condition for the Loewner equation to generate slits, to appear in Ann. Acad. Sci. Fenn.

[13] G. Lawler, O. Schramm, W. Werner, Values of Brownian intersection exponents I: Half-plane exponents, Acta Math. 187 (2001), 237-273. MR.1879850(2002m:60159a)

[14] G. Lawler, O. Schramm, W. Werner The dimension of the planar Brownian frontier is 4/3, Math. Res. Lett. 8 (2001), 401-411. MR1849257(2003a:60127b)

[15] O. Lehto, K. Virtanen, Quasiconformal mappings in the plane, Springer 1973. MR0344463 $(49: 9202)$

[16] D. E. Marshall, S. Rohde, Convergence of the Zipper algorithm for conformal mapping, preprint available at http://www.math.washington.edu/ marshall/preprints/preprints.html

[17] Chr. Pommerenke, Univalent Functions, Vandenhoeck and Ruprecht, Göttingen (1975). MR0507768 (58:22526)

[18] Chr. Pommerenke, Boundary behaviour of conformal maps, Springer (1992). MR.1217706 (95b:30008)

[19] S. Rohde, O. Schramm, Basic properties of SLE, arXiv:math.PR/0106036, to appear in Ann. Math.

[20] O. Schramm, Scaling limits of loop-erased random walks and uniform spanning trees, Israel Jour. Math. 118 (2000), 221-288. MR1776084 (2001m:60227)

[21] S. Smirnov, Critical percolation in the plane: conformal invariance, Cardy's formula, scaling limits, C. R. Acad. Sci. Paris Ser. I Math. 333 (2001), 239-244. MR1851632 (2002f:60193)

Department of Mathematics, University of Washington, Seattle, Washington 981954350

E-mail address: marshall@math.washington.edu

Department of Mathematics, University of Washington, Seattle, Washington 981954350

E-mail address: rohde@math.washington.edu 